(Aus der Universitäts-Kinderklinik Würzburg.)

\title{
Die pathogenetische Bedeutung der Fettsäuren in Fettmileh-Nahrungen.
}

(Ein Beitrag zur Frage der, ,zersetzten Milch".)

Von

Prof. Dr. Rietschel-Würzburg.

(Eingegangen am 3. Dezember 1920.)

I. Experimentellex Teil

in Gemeinschaft mit den Herren Dr. Kuhn und Zimmermann.

Gerade in der jetzigen Zeit der Milchknappheit wird das Problem der bakteriell zersetzten Milch wieder akut. Zwei Anschauungen sind dabei auch heute noch vorherrschend. Einmal werden durch Saprophyten $^{1}$ ) gebildete toxische Produkte, die giftig wirken, schuldig gemacht und sodann hat man vorzugsweise an Säuren gedacht, speziell niedere Fettsäuren (Czern y und seine Schule), die in der verdorbenen Milch dem Kinde schädlich sein könnten. Eine ausführliche Kritik der ersten Hypothese muß ich unterlassen, zumal wir keine neuen experimentellen und klinischen Beweise gegen sie beibringen können. Theoretisch, experimentell und klinisch ist aber diese Hypothese sehr wenig gestützt. Ich verweise auf meine früheren Arbeiten in dieser Frage ${ }^{2}$ ).

Anders steht es mit der zweiten Anschauung, daß in der Milch vor dem Genuß freie Fettsäuren sich bilden können, die schädliche Wirkungen ausüben. Diese Theorie ist in erster Linie von Czerny und Keller aufgestellt, die allerdings, das sei betont, nicht allein die exogen entstandenen Säuren verantwortlich machen wollen, sondern auch eine endogene Entstehung dieser Säuren aus Zucker und Fett annchren. Im wesentlichen halten sie aber an dem Begriff der ,, bakteriell zersetzten Milch" fest und verstehen darunter alle jene Milchzersetzungen, in der durch Bakterienwirkung vor dem Genuß besonders aus dem Fett eine

1) DaB Infektionen der Milch durch echte pathogene Keime, insbesondere Typhus, Paratyphus, shwere Erkrankungen nach sich ziehen, ja Endemien hervorrufen können, daran ist nicht im geringsten $\mathrm{zu}$ zweifeln, doch sind solche Endemien etwas sehr Seltenes.

2) Rietschel, Ergebn. d. Innern Med. u. Kinderheilk. 6 und Münch. med. Wochenschr. 1920, Nr. 2. 
freie Fettsäurebildung stattgefunden hat. Czerny hat dies erst kürzlich bei der Empfehlung seiner Einbrennenahrung wieder klar betont und Birk ${ }^{1}$ ), ein Schüler Czernys, sagt in der neuesten Auflage (1920) seines Leitfadens: „Die akuten Ernährungsstörungen kommen dadurch zustande ${ }^{2}$ ), daß die Nahrung der Kinder schon vor der Verabreichung gewissen bakteriellen Zersetzungen anheimfällt. Das Ergebnis der Zersetzung sind Gärungsfettsäuren, die, wie auch experimentell festgestellt ist, den Durchfall und die ihn begleitenden Symptome heraufführen können".

Die Sommerhitze meint er, begünstigt dies, indem sie ,das Bakterienwachstum und damit die Milchverderbnis befördert. Doch ist der Einfluß der Bakterien kein direkter. Es handelt sich nicht um Toxine..., sondern das schädliche Agens stellen die aus den Nahrungsbestandteilen gebildeten bereits erwähnten Zersetzungsprodukte der Bakterien ,die Fettsäuren " dar".

Die ganze Säurefrage und ihre pathogenetische Bedeutung für die Entstehung von Durchfällen hat durch eine größere Zahl von Arbeiten Langsteins und seiner Schüler aus dem Kaiserin Auguste ViktoriaHaus ${ }^{3}$ ) eine Bearbeitung erfahren, die kurz gesagt, zu dem Resultat kommen, daß den geringen Mengen freier Fettsäuren, die sich exogen in der Nahrung vor dem Trinken finden, keine pathogenetische Bedeutung zugesprochen werden kann. In gewöhnlichen frischen Kuhmilchmischungen. finden sich überhaupt keine freien Fettsäuren und die Mengen, die im Magen zur Abspaltung gelangen, sind viel zu gering, um pathogenetisch für die akuten Ernährungsstörungen in Betracht zu kommen.

Die größten Mengen freier Fettsäuren fanden Bahrdt und Edelstein in der holländischen Säuglingsnahrung, und zwar Mengen, die etwa 4-9 $\mathrm{cm}^{\mathrm{n}} / 10 \mathrm{NaOH}$ auf $100 \mathrm{~g}$ Nahrung entsprechen, also auf Essigsäure berechnet, $0,025-0,054 \mathrm{~g}$. Daß diese Mengen nicht schädlich sein können, beweisen einmal die Erfolge mit der Buttermilch und sodann ausgedehnte Tierversuche. In keiner anderen Nahrung aber, und selbst in solcher, die mehrere Tage im Brutschrank stand, konnten nennenswerte Mengen flüchtiger Fettsäuren gefunden werden und auch dort, wo es möglich war, die Milch zu untersuchen, durch deren Genuß angeblich Kinder schwer toxisch geworden waren, konnten nie größere Mengen solcher freien Fettsäuren festgestellt werden. Auch die klinischen

1) Birk, Leitfaden der Kinderheilkunde, I. Teil. 4. Aufl. 1920, S. 113.

2) In der ersten Auflage 1914 fügt Birk noch hinzu: ,kommen weitaus am häufigsten dadurch zustande".

3) Die gesamten Arbeiten darüber finden sich in der Zeitschrift für Kinderheilkunde in den Bänden $1,3,4,5$ und 11. Die Arbeiten sind in Band 11 dieser Zeitschrift S. $403 \mathrm{ff}$. aufgezeichnet. 
Beobachtungen mit gesäuerter Milch sprechen weitaus mehr dafür, daß die gewöhnlich gesäuerte Milch umbedenklich zur Fütterung verwendet werden konnte. Ich erwähne einmal die Beobachtungen von mir, Nebeli), Bernheim-Karrer ${ }^{2}$ ) und erst jüngst hat wieder $\mathrm{Hamburger}^{3}$ ) darauf hingewiesen, leicht angesäuerte Milch als Nahrungsmittel für die Kinder ruhig zu verwenden. Insbesondere sind es aber die vielen Erfahrungen mit Buttermilch, in der zweifellos geringe Menge Fettsäuren enthalten sind, die gegen die Ansicht von der pathogenetischen Bedeutung der in der Milch exogen gebildeten freien Fettsäuren sprechen.

Nun ist aber die ganze Frage einmal durch die Einführung der Buttermehlnahrung von Czerny-Kleinschmidt sowie durch klinische Beobachtungen Berend $\mathbf{s}^{4}$ ) wieder in den Vordergrund gerückt. Czerny - Kleinschmidt betonen zwar ausdrücklich bei der Besprechung der Erfolge ihrer Nahrung, daß sie den Erfolg nicht einer einzigen Ursache zuschreiben möchten, daß sie es aber für ganz besonders günstig ansehen, daß bei der Buttermehlnahrung durch den Einbrenneprozeß die Butter von den flüchtigen Fettsäuren befreit wird, die für Kinder schädlich wirken. Und Berend führt in der Tat beachtenswerte klinische Beobachtungen an, die diese Theorie zu stützen vermögen. Auch Thiemich ${ }^{5}$ ) hat jüngst in einer Kritik zu unseren Arbeiten Stellung genommen und meint, ,daß die Frage keineswegs spruchreif ist". So ist es es wohl am Platze, auf diese Dinge näher einzugehen.

Wir haben versucht auf experimentellem und klinischem Wege der Frage näherzutreten. Zunächst war einmal dabei festzustellen, ob denn wirklich in der Buttermehlnahrung, auch wenn man reine Butter ohne Einbrenne zugibt, viele freie Fettsäuren enthalten sind. Und sodann haben wir die Buttermehlnahrung mit und ohne Einbrenneprozeß 24 Stunden stehengelassen, oft während des warmen'Sommers, bis sie völlig gesäuert war (meist betrug dieser Säuregrad um 20 HenkelSoxhlet) und dann verfüttert. Sollten wirklich aus dem Fett viele solche Säuren entstehen, so müßte der Säuerung dieser Fettnahrung besondere Bedeutung zukommen. Stets wurde gekochte Milch verwandt, da es uns hier nur auf die Bestimmung der angeblich schädlichen Festtsäuren ankam. Entweder wurde die Milch gleich gekocht, um dann später zu sauern, oder die Milch wurde zuerst als Butternahrung znbereitet und vor dem Trinken aufgekocht. Ähnlich wie Huldschins $\mathrm{ky}^{6}$ ) dies getan hat, haben wir alsdann einer größeren

1) Rietschel, 1. c. Nebel, Jahrbuch f. Kinderheilk. 63, 752.

2) Bernheim - Karrer, Zeitschr. f. Kinderheilk. 13, 435.

3) Hamburger, Münch. med. Wochenschr. 1919, Nr. 21.

4) Berend, Monatsschr. f. Kinderheilkd. 14, 601 .

5) Thiemich, Beiheft zur med. Klinik 1920.

$\left.{ }^{6}\right) \mathrm{Huldschinsky}$, Zeitschr. f. Kinderheilkde 3, 366. 
Anzahl von Kindern nach $11 / 2$ bis 2 Stunden den Magen ausgehebert und den Mageninhalt auf freie und gebundene Fettsäuren untersucht, ob es vielleicht hier möglich wäre, durch eine Buttermehlnahrung, sei sie süß oder saver, sei sie mit oder ohne Einbrenne hergestellt, sebr viel höhere Fettsäurewerte zu finden, die pathogenetisch für Störungen der Verdauung verantwortlich gemacht werden könnten. Die Methodik, deren wir uns bedienten, war die gleiche von Bahrdt, Langstein, Edelstein und $W_{\text {elde }}{ }^{1}$ ) angegebene. Die Säuren wurden im Vakuum mit Dampf destilliert; um nicht weitschweifig zu werden, wollen wir hier nur auf das Verfahren verweisen. Wir haben die Methode nur schätzen gelernt ${ }^{2}$ ).

Die Mengen von Fettsäuren, die wir zunächst in verschiedenen Nahrungsgemischen vor dem Trinken fanden (Fettmilch, Kellers Malzsuppe usw.) waren sehr gering. Das gleiche hatten schon Langstein und seine Mitarbeiter gefunden. Es erübrigt sich, sie daher ausführlich mitzuteilen. Für die Buttermehlnahrung, sei sie mit oder ohne Einbrenne hergestellt, ergaben sich folgende Werte:

Tabelle 1. Bestimmung der flüchtigen Fettsäuren vor der Nahrungsverfutterung.

\begin{tabular}{|c|c|c|c|}
\hline \multirow[b]{2}{*}{ Nahrung $100 \mathrm{cem}$} & \multirow{2}{*}{$\begin{array}{c}\text { Gesamtacidităt } \\
\frac{\mathrm{n}}{10} \mathrm{NaOH}\end{array}$} & \multicolumn{2}{|c|}{ Flüchtige Fettsăuren } \\
\hline & & $\begin{array}{l}\text { o } 110 \mathrm{Na}(\mathrm{OH}) \text { auf } 100 \mathrm{ccm} \\
\text { Nanrungsmenge }\end{array}$ & als Essigsäure berechnet \\
\hline $\begin{array}{l}\text { Süße Buttermehlnahrung } \\
\text { nach Czerny-Klein- } \\
\text { schmidt }\end{array}$ & 5,1 & 0,3 & 0,0018 \\
\hline $\begin{array}{l}\text { Süße Buttermehlnahrung } \\
\text { ohne Finbrenne }\end{array}$ & $\begin{array}{l}4,4 \\
6,5\end{array}$ & $\begin{array}{l}0,3 \\
0,4\end{array}$ & $\begin{array}{l}0,0018 \\
0,0024\end{array}$ \\
\hline
\end{tabular}

Aus dieser Tabelle ist zu ersehen, daß in der gewöhnlichen Buttermehlnahrung so gut wie keine freien Fettsäuren vorhanden sind, sei sie nach der Methode von Czerny-Kleinsohmidt, sei sie ohne Einbrenne hergestellt; in beiden Fällen enthält sie fast gleiche Mengen freier Fettsäuren. Es fehlen hier selbstverständlich nur die freien und gebundenen flüchtigen Fettsäuren, nicht die Glyceride derselben. Auf sie wird später noch bei der Magenausheberung zurückzukommen sein.

In allen sauren Nahrungen dagegen findet sich stets ein gewisser Anteil, der auf flüchtige Fettsäuren zu beziehen ist, wenn auch der weitaus größere Anteil der Säure auf die Milchsäure kommt. Diese flüchtigen Fettsäuren stammen aber aus der bakteriellen Milchzuckergärung,

1) Bahrdt, Langstein, Edelstein, Welde, Zeitschr. f. Kinderheilk. 1, 139 ,

2) Die Untersuchungen wurdeu teils im Hygienisehen Institut (Prof: K. B. Lehmann), teils im Pharmakologischen Institut (Prof. Flury) ausgeführt. Beiden Herren sei auch an dieser Stelle der Dank ausgesprochen. 
Die pathogenetische Bedeutung der Fettsăuren in Fettmilch-Nahrungen. 187

und zwar werden neben der Milchsäure einzelne freie flüchtige Fettsäuren, besonders geringe Mengen Essigsäure gebildet. Die Menge dieser Substanzen hängt einmal von der zeitlichen Einwirkung der Bakterien $a b$ und sodann natürlich von der Art der verschiedenen Gärungserreger. Die gewöhnlichen Milchsäurebacillen (Streptococcus Güntheri) lassen wohl nur geringe. Mengen dieser Säuren entstehen, speziell die Essigsäure.

Die folgende Tabelle gibt die fï̈chtigen Fettsäuremengen in saurer Milch wieder.

Tabelle 2. Gesauerte Milchnahrungen.

\begin{tabular}{|c|c|c|c|c|}
\hline & & $\begin{array}{l}\text { Flüchtige } \\
\text { in } \begin{array}{l}1 / 10 \mathrm{NaOH} \\
\text { in } 100 \mathrm{~g}\end{array}\end{array}$ & $\begin{array}{c}\text { Fettsäuren } \\
\text { in } 100 \mathrm{~g} \\
\text { als Essigsäure } \\
\text { berechnet } \\
\mathrm{g}\end{array}$ & $\begin{array}{l}\text { Gesamt- } \\
\text { acidität } \\
\text { Henkel- } \\
\text { Soxhlet }\end{array}$ \\
\hline $\left.1^{1}\right)$. & Kuhmilch, 2 Tage gesäuert. & 3,8 & 0,023 & _- \\
\hline $\left.2^{1}\right)$. & Bei Sommerhitze 10 Tage. . & 8,3 & 0,05 & 一 \\
\hline $\left.3^{1}\right)$. & Bei Sommerhitze . . . . & 4,5 & 0,027 & - \\
\hline 4. & Buttermilch . . . . . & 2,0 & 0,012 & 23,0 \\
\hline 5. & $\begin{array}{l}\text { Buttermehlnahrung nach } \mathrm{Czerny} \text { - } \\
\text { Kleinschmidt } 24 \text { Std. bei }\end{array}$ & & & \\
\hline & Wärme stehen gelassen . . . & 1,1 & 0,0066 & 19,8 \\
\hline 6. & Desgleichen . . . . . . . . . & 1,5 & 0,009 & 16,4 \\
\hline 7. & Desgleichen . & 2,15 & 0,019 & 13,34 \\
\hline 8. & Buttermehlnahrung, 24 Std. stehen & & & \\
\hline & gelassen, sauer, ohne Einbrenne & 1,3 & 0,0078 & $.16,4$ \\
\hline 9. & Desgleichen .......... & 1,7 & 0,0102 & 16,2 \\
\hline 10. & Desgleichen . . . . . & 1,75 & 0,0105 & 18,2 \\
\hline 11. & Desgleichen . . . . . & 2,6 & 0,0156 & 17,0 \\
\hline
\end{tabular}

Aus diesen Werten geht hervor, daß selbst in der sauren Buttermehlnahrung, die ohne Einbrenne hergestellt ist, nicht wesentlich mehr freie flüchtige Fettsäuren enthalten sind als in der gewöhnlichen Buttermilch, und daß eine Entstehung der Säuren aus dem Fett durch die Säuerung vor dem GenuB nicht statthat. Das scheint uns aber von ausschlaggebender Bedeutung.

Sind diese geringen Mengen Säuren nun gleichgültig oder schädlich ? Darauf hat bis zu einem gewissen Grade die klinische Erfahrung mit Buttermilch schon die Antwort gegeben, dà $\beta$ in diesen geringen Mengen, in der sie in der gewöhnlichen Buttermilch vorkommen, eine Schädigung des Kindes durch die Anwesenheit dieser Säuren, ebensowohl der Milchsäure wie der anderen Säuren nicht nachgewiesen ist, zumindest nicht beim gesunden Kinde. Auch beim gesunden Brustkind entstehen ja aus einem Teil des mit der Frauenmileh eingeführten Milchzuckers

1) Nach Bahrdt-Edelstein. 
durch Vergärung Säuren und wohl auch neben der Milchsäure geringe Mengen flüchtige Fettsäuren (allerdings meist wohl erst in den tieferen Teilen des Darms, besonders also im Dickdarm), - ja wir dürfen wohl annehmen, daß diese Säuerung physiologisch ist und für den physiologischen Nutzungseffekt der Frauenmilch von Bedeutung ist. Auch im Magen können geringe Mengen von Milchsäure sich bilden. Das, was die Frauenmilch aber auszeichnet, ist nur, daß diese Säuerung im Darm stets „in physiologisehen Grenzen“ bleibt. Eine geringe Menge Milchsäure scheint auch bei Kuhmilchernährung günstig zu wirken, ja direkt von Vorteil zu sein, worauf Klotz' ${ }^{1}$ ) in seinen bekannten Stoffwechseluntersuchungen hingewiesen hat. Freilich scheint es, da $B$ beim lkünstlich genährten Kind viel leichter ,diese physiologische Grenze" über-

Tabelle 3.

\begin{tabular}{|c|c|c|c|c|}
\hline Mageninhult bei & $\begin{array}{c}\text { Ver- } \\
\text { suchs- } \\
\text { Nr. }\end{array}$ & $\begin{array}{c}\text { Ausgehebert } \\
\text { nach } \\
\text { Stunden }\end{array}$ & $\begin{array}{c}\mathrm{cem} n / 10 \mathrm{Na}(\mathrm{OH}) \\
\text { auf } 100 \mathrm{ccm} \\
\text { Mageninhalt } \\
\end{array}$ & $\begin{array}{c}\text { Als Fssigsäure } \\
\text { berechnet } \\
\mathrm{g}\end{array}$ \\
\hline \multirow{6}{*}{$\begin{array}{l}\text { süBer Buttermehlnahrung } \\
\text { nach Ozerny-Klein- } \\
\text { schmidt }\end{array}$} & 1 & $1^{3} / 4$ & 9,91 & 0,060 \\
\hline & 2 & $21 / 4$ & 23,04 & 0,138 \\
\hline & 3 & $11 / 2$ & 16,24 & 0,097 \\
\hline & 4 & $1 / 2$ & 12,54 & 0,095 \\
\hline & 5 & $11 / 2$ & 13,8 & 0,083 \\
\hline & 6 & $1^{3} / 4$ & 16,45 & 0,099 \\
\hline \multirow{7}{*}{ ohne Einbrenne hergestellt } & I & $13 / 4$ & 17,2 & 0,103 \\
\hline & 2 & 2 & 21,1 & 0,127 \\
\hline & 3 & $11 / 2$ & 11,01 & 0,066 \\
\hline & 4 & $11 / 2$ & 10,83 & 0,065 \\
\hline & 5 & $11 / 2$ & 10,8 & 0,065 \\
\hline & 6 & 2 & 12,76 & 0,076 \\
\hline & 7 & 2 & 9,64 & 0,058 \\
\hline
\end{tabular}

schritten wird, und daß dann pathologische Reize an ihre Stelle treten, d. h. es kommt wohl viel schneller zu einer endogenen Infektion oder Invasion des sterilen Darms und damit zu abnormer Säurebildung. Es ist gewiß ein nicht hoch genug anzuschlagendes Verdienst Czern ys, die große Rolle erkannt zu haben, die organische Säuren bei der Pathogenese akuter Verdauungsstörungen spielen. Nur sind wir der Meinung, daß die schädliche Wirkung im wesentlichen von einer endogenen Entstehung dieser Säuren im Darm abhängt und daß den exogen vor dem Trinken gebildeten flüchtigen Fettsäuren, deren Menge viel zu gering ist, tatsächlich keine pathogenetische Bedeutung zukommt.

Wir haben alsdann untersucht, wie sich im Magen die verschieden hergestellten Arten der Buttermehlnahrung verhalten und ob viel Fettsäuren entstehen, besonders bei den sauren Fettnahrungen. Solche Untersuchungen hat mit gewöhnlichen Nahrungen in ausgedehntem

1) Klotz, Milchsäure und Stoffwechsel. Jahrb. f. Kinderheilk. $\mathbf{0}$, 1. 
Maße Huldschinsky ausgeführt. Er fand, daß diese Säuren den im Milchfett präformierten Säuren entsprechen, daß sie aber lediglich durch fermentative Spaltung der Glyceride zu erklären sind. Wir lassen zunächst die Untersuchungen mit süßer Buttermehlnahrung folgen. Wir bemerken dabei, daß die ersten Untersuchungen mit einer Buttermehlnahrung ausgeführt werden, die nach Czerny-Keller hergestellt waren, während später bei uns einfach die Butter der Milchmehlnahrung während des Abkühlens zugesetzt und durch Schlagen verteilt wurde.

Aus nebenstehender Tabelle 3 geht nun hervor, daß die Zahlen, die bei der Magenuntersuchung der mit Czern y-Klein sch midt hergestellten Buttermehlnahrung genährten Kinder im großen und ganzen fast die gleichen Durchschnittswerte ergaben, wie die ohne Einbrenne hergestellten, m. a. W., daß die geringen Mengen flüchtiger Fettsäuren, die bei der Einbrenne verlorengehen, so gut wie irrelevant sind. Wir sind dann dazu übergegangen, die ohne Ein brenne (also durch bloßen Zusatz von Butter) angereicherte Buttermehlnahrung 24 Stunden stehenzulassen und sie nach Verfütterung auf den Gehalt von freien Fettsäuren zu untersuchen. Wir erhielten dabei folgende Werte. Die Ausheberung geschah gewöhnlich nach 2 Stunden (Tab. 4).

Tabelle 4.

\begin{tabular}{|c|c|c|c|c|}
\hline Mageninhalt bei & $\begin{array}{l}\text { Ver. } \\
\text { suchs- } \\
\text { Nr. }\end{array}$ & $\begin{array}{l}\text { Ausgehebert } \\
\text { nach } \\
\text { Stunden }\end{array}$ & $\begin{array}{l}\text { ccm n/10 Na(OH) } \\
\text { auf } 100 \mathrm{ccm} \\
\text { Mageninhalt }\end{array}$ & $\begin{array}{c}\text { Als Essigsäure } \\
\text { berechnet } \\
\text { g }\end{array}$ \\
\hline saurer Buttermehlnahrung & $\begin{array}{r}1 \\
2 \\
3 \\
4 \\
5 \\
6 \\
7 \\
8 \\
9 \\
10 \\
11 \\
12 \\
13 \\
14\end{array}$ & $\begin{array}{l}2 \\
2 \\
2^{1 / 4} \\
2^{1 / 4} \\
2^{1 / 4} \\
2^{1} / 4 \\
2^{1 / 4} \\
1^{1 / 4} \\
11 / 2 \\
2 \\
2 \\
2 \\
2 \\
2\end{array}$ & $\begin{array}{l}13,74 \\
13,79 \\
26,6 \\
23,1 \\
25,8 \\
23,4 \\
18,44 \\
7,65 \\
7,97 \\
10,38 \\
12,64 \\
10,74 \\
13,33 \\
15,9\end{array}$ & $\left.\begin{array}{l}0,082 \\
0,083 \\
0,160 \\
0,139 \\
0,151 \\
0,140 \\
0,110 \\
0,046 \\
0,047 \\
0,062 \\
0,076 \\
0,064 \\
0,080 \\
0,096\end{array}\right\} 0,096$ \\
\hline
\end{tabular}

Aus dieser Tabelle geht hervor, daß die Mengen der flüchtigen Fettsäuren bei der mit Butter angereicherten und sauer gewordenen Nahrung relativ gering ist. Gewiß sind, was nicht verwunderlich ist, die Mengen bei saurer Milch etwas höher, wenn wir von den Versuchen 8 und 9 absehen, wo nach $1 \frac{1}{2}$ Stunden ausgehebert wurde. Doch ist es erstaunlich, wie gering dieser Unterschied ist. 
Bahrdt und seine Mitarbeiter haben festgestellt, da $B$ im Magen hauptsächlich Buttersäure und weniger andere Fettsäuren gebildet werden. Daß diese im Magen sich bildenden Säuren durch fermentative Spaltungen der Glyceride entstehen, scheint uns ebenso wie Bahrdt wahrscheinlich. Ob daneben nicht auch bakterielle Prozesse nebenher gehen, aber immerhin möglich. Immerhin ist es uns wie Bahrdt aufgefallen, daß fettfreie oder fettarme Gemische keine wesentliche Erhöhung der freien. Fettsäuren im Magen zeigen wie vor dem Genuß. Wir verzichten darauf, die Ergebnisse mitzuteilen, da sie mit.denen von Bahrdt und seinen Mitarbeitern gefundenen übereinstimmen. Aber das geht auch aus unseren vorher mitgeteilten Untersuchungen bei Butternahrungen hervor, da $\beta$ die Mengen

Tabelle 5.

\begin{tabular}{|c|c|c|c|c|}
\hline Mageninhalt bei & $\begin{array}{l}\text { Ver- } \\
\text { suchs- } \\
\text { Nr. }\end{array}$ & $\begin{array}{l}\text { Ausgehoben } \\
\text { nach } \\
\text { Stunden }\end{array}$ & $\begin{array}{l}\text { cam } \mathrm{n} / 10 \mathrm{Na}(\mathrm{OH}) \\
\text { auf } 100 \mathrm{ccm} \text { Magerı } \\
\text { inhalt }\end{array}$ & $\begin{array}{c}\text { Als Essigsãure } \\
\text { berechnet } \\
\text { g }\end{array}$ \\
\hline \multirow{6}{*}{ süßer Margarinemehlnahrung } & 1 & 2 & 5,2 & 0,03 \\
\hline & 2 & 2 & 13,1 & 0,079 \\
\hline & 3 & 2 & 6,0 & 0,037 \\
\hline & 4 & 2 & 15,8 & 0,096 \\
\hline & 5 & 2 & 14,3 & 0,086 \\
\hline & 6 & 2 & 18,6 & 0,112 \\
\hline \multirow{5}{*}{ saurer Margarinenahrung } & 1 & 2 & 12,8 & 0,078 \\
\hline & 2 & 2 & 19,1 & 0,114 \\
\hline & 3 & 2 & 22,5 & 0,134 \\
\hline & 4 & 2 & 10,8 & 0,065 \\
\hline & 5 & $11 / 4$ & 9,5 & 0,057 \\
\hline
\end{tabular}

der im Magen sich bildenden flüchtigen Fettsäuren, die zumeist wohl aus dem Fett abgespalten werden, nicht wesentlich dadurch vermehrt werden, ob man vor dem Genuß die Butter über dem Feuer erhitzt, oder ob sie gleich so der Nahrung zugesetzt wird. Ja selbst in einer solchen sauren Nahrung sind diese Fettsäuren im Magen nicht allzusehr vermehrt, da eben bei der Säuerung vor dem Genuß der Milch) sich nur ganz geringe Mengen flüchtiger Fettsäuren bilden, und diese auch nur aus dem Zucker, aber nicht aus dem Fett stammen. Unsere Versuche machen daher eine pathogenetische Bedeutung dieser vor dem Genuß entstandenen Säuren nicht nur unwahrscheinlich, sondern schließen sie aus. Die tatsächliche Menge dieser Säuren, die sich in der Milch exogen vor dem Genuß bilden, ist eben sehr gering. Und nach allem, was wir wissen, sind diese geringen Mengen nicht imstande, bei der Entstehung von Verdauungsstörungen eine wirkliche größere Rolle 
zu spielen. Selbst aber die Mengen, die im Magen abgespaltet werden, sind noch äußerst gering. Daß unter pathologischen Verhältnissen aus der Nahrung solche Säuren im Darm bei endogener Infektion entstehen können, soll nicht geleugnet werden, aber die exogen mit der Nahrung (sei sie süß oder sauer, sei die Butter gebräunt oder nicht gebräunt zugesetzt) verabreichten Säuren sind für das Kind irrelevant.

Wir haben auch untersucht, ob in einer Mehifettnahrung, die statt der Butter Margarine enthält, vielleicht wesentlich weniger flüchtige Fettsäuren enthalten sind. (Siehe nebenstehende Tabelle 5.)

Die Zahlen unterscheiden sich nicht wesentlich von denen bei Buttermehlnahrung, trotzdem man eher erwarten sollte, da $B$ sie geringer sein würden, da der Margarine Glyceride von flüchtigen Fettsäuren im allgemeinen fehlen. Dies deutet doch darauf hin, daß vielleicht auch bakterielle Spaltungen neben fermentativen schon im Magen eine Rolle spielen könnten.

\section{Klinischer Teil.}

Es lag nahe, neben den experimentellen Erfahrungen auf Fettsäuren auch klinische Erfahrungen zu sammeln, wie solche saure Fettnahrung von jungen Säuglingen vertragen würde. Wir haben ausgedehnte Untersuchungen an gesunden und kranken Kindern angestellt und dürfen als absolut feststehendes Resultat vorwegnehmen, daB wir niemals den geringsten Schaden von gesäuerter Milch und insbesondere nicht vonder sauren Buttermehlnahrung gesehen haben, sei sie mit oderohne Einbrenne hergestellt. Wir haben dies schon in einer früheren Arbeit in der Münchener medizinischen Wochensohrift mitgeteilt, haben aber noch weitere Erfahrungen gesammelt, die aber nur unsere bisherigen Erfahrungen bestätigen können, ja wir konnten sogar öfter uns des Eindrucks nicht erwehren, als ob die saure Buttermehlnahrung oft besseren Ansatz zur Folge hatte. Leider erlaubt es die Knappheit der Papierverhältnisse nicht, die Krankengeschichten in Kurven oder Tabellen zu veröffentlichen. Ich tue es daher nur in einer zusammengedrängten Übersicht und weiß wohl, daß dies nicht so überzeugend ist wie eine kurvenmäßige Darstellung. Einen großen Teil der Kurven habe ich zeichnen lassen. Sie stehen für jeden, der sich dafür interessiert, zur Verfügung.

Klinisch wurde dabei folgendermaßen verfahren: Die Milch bezogen wir von einer großen Molkerei, die im wesentlichen ihr Milchquantum durch Fuhrwerk oder Bahn vom Land hereinbekam. Besondere Sorgfalt war bei der Gewinnung der Milch nicht beobachtet worden, wie ja heute der Begriff der Kindermilch und Vorzugsmilch ein völlig hinfälliger geworden ist und dadurch doch nirgends, wie es scheint, 
Schaden gestiftet worden ist ${ }^{1}$ ). Die Nahrung wurde trinkfertig zubereitet, ohne daß die Milch gekocht wurde. Am Anfang wurde die Butter als Einbrenne hinzugetan, später taten wir dies nicht mehr, sondern es wurden eintach Butter und Mehl verrührt und dann diese Buttermehlnahrung 24 Stunden stehengelassen. Nur das Mehl wurde mit Wasser vorher einige Minuten gekocht. Nach 24 Stunden, manchmal nach etwas längerer Zeit, wurde der Säuregrad bestimmt, der in der Tabelle nicht einzeln mit aufgeführt ist und der etwa um 20 Soxhlet-Henkel-Grade schwankt. Die Milch wurde meist kurz aufgekocht, um die Tuberkelbacillen abzutöten, sei es vor dem Trinken, sei es bei der Einlieferung, zumal ja auch in erster Linie nur auf die Schädlichkeit der Säuren Rücksicht genommen werden sollte. Die Buttermehlnahrung wurde so zubereitet, daß sie als Halbmilch gegeben wurde mit 3\% Butter, 3\% Mehl und 5\% Zucker und etwas Salz.

Leider war es nicht möglich, Kurven oder auch nur Tabellen zu bringen. Wir haben daher möglichste Kürzung vorgenommen.

Gut gedeihende Kinder bei saurer Buttermehlnahrung. (Die beiden Anfangsbuchstaben bedeuten den Namen, dann kommt das Alter.) $A=$ das Anfangsgewicht, bei dem die Fütterung mit der sauren Nahrung begonnen wurde.

B = Zahl der Tage, während die Nahrung gegeben wurde. (Beobachtungsdauer).

$\mathbf{E}=$ Endgewioht bei Absetzen von der sauren Buttermehlnahrung.

T.Z. = Durchschnittliche tägliche Zunahme in Gramm während.dieser Ernährung. s.B.M.N = Saure Buttermehlnahrung.

$\begin{array}{llll}\text { 1. R. L. } 14 \mathrm{Tg} . & \text { A } 2260 \mathrm{~g} & \text { B } 30 \text { Tage. E } 3250 & \text { T.Z. } 33 \mathrm{~g} \text {. }\end{array}$ Rekonvaleszent nach enteralem Infekt.

$\begin{array}{llllll}\text { 2. Sch. H. } 2 \frac{1}{2} \text { Mon. A } 3640 \mathrm{~g} & \text { B } 33 \text { Tage. E } 4380 & \text { T.Z. } 29 \mathrm{~g} \text {. }\end{array}$ Rekonvaleszent nach enteralem Infelst.

3. W. H. 3 Mon. A $5260 \mathrm{~g}$ B 10 Tage E 5550 T.Z. $29 \mathrm{~g}$ Bilanzstörung, unterbrochen durch eine fieberhafte Grippe. ('T. 38,9.)

$\begin{array}{llllll}\text { 4. Chr. A. } 3 \text { Mon. A } 5190 & \text { B } 29 \text { Tage } & \text { E } 5560 & \text { T.Z. } 13 \mathrm{~g}\end{array}$ Parentaler Infekt, Bilanzstörung.

$\begin{array}{llllll}\text { 5. R. Ph. } 2 \text { Mon. A } 2590 & \text { B } 20 \text { Tage } & \text { E } 3260 & \text { T.Z. } 34 \mathrm{~g}\end{array}$ Reparation nach akuter Störung.

$\begin{array}{llllll}\text { 6. H. N. } 9 \text { Mon. A } 3940 & \text { B } 9 \text { Tage } & \text { E } 4300 & \text { T.Z. } 40 \mathrm{~g}\end{array}$ Kein Gedeihen bei Vollmilch, bei s.B.M.N. gute Zunahme. 7. A. H. $\quad \underset{\text { Mon, }}{\text { A } 3820} \underset{\text { Reparation nach }}{\text { B } 24 \text { Tage }} \underset{\text { akuter Dyspepsie. }}{\text { E } 4400} \quad$ T.Z. $24 \mathrm{~g}$

1) Auch das sollte uns recht stutzig machen, daß kaum einem Säugling mehr sog. Kindermilch verfüttert wird und jede Mutter nur froh ist, wenn sie Milch erhält und nicht fragt, wie sie gewonnen ist. Trotzdem sehen wir im allgemeinen keine Erhöhung der Sterblichkeit, auch nicht im Sommer. Schloss mann spricht sogar vom Verschwinden des Sommergipfels während des Krieges. 

$\begin{array}{llllll}\text { 8. L. E. } 1 \frac{1}{2} \text { Mon. A } 3350 & \text { B } 16 \text { Tage E } 3820 & \text { T.Z. } 30 \mathrm{~g}\end{array}$ Reparationsstadium nach akuter Dyspepsie.
$\begin{array}{llllll}\text { 9. R. M. } 5 \text { Mon. A } 5120 & \text { B } 24 \text { Tage } & \text { E } 5680 & \text { T.Z. } 23 \mathrm{~g}\end{array}$ Hydrocephalus, daneben parenterale Infektion.

10. R. G. 5 Mon. A 5660 B 21 Tage E 6560 Bilanzstörung nach parenteraler Infektion.

11. L. F.

5 Mon. A 4680

B 13 Tage E 5220

T.Z. $43 \mathrm{~g}$ Pylorospasmus und Rekonvaleszenz.

12. A. G.

2 Mon. A 3180

B 24 Tage E 3620

T.Z. $18 \mathrm{~g}$ Rekonvaleszent nach parenteralem Infekt.

13. M. W. 2 Mon

A 3420

B 16 Tage E 4000

T.Z. $36 \mathrm{~g}$ Bilanzstörung, Hypotrophie.

14. L. K. 21/2 Mon. A 4600 B 15 Tage E 5100

T.Z. $33 \mathrm{~g}$ Dystrophie nach parenteralem Infekt.

15. Seh. B. 6 Mon.

A $3450 \quad$ B 20 Tage E 4200

T.Z. $37 \mathrm{~g}$ Dystrophie nach Infekt.

16. H. G. 5 Mon. A $3800 \quad$ B 43 Tage E 4600 Leicht gestörtes Kind (Bilanzstörung).

17. K. R. 7 Mon. A 4000 B 22 Tage E 4300

Pertussis. Chinin. Allmähliche Besserung.

18. F. D. 9 Mon. A $4800 \quad$ B 24 Tage E $5600 \quad$ T.Z. $33 \mathrm{~g}$ Pertussis.

19. H. W. 5 Mon. A 4200 B 24 Tage E $4900 \quad$ T.Z. $29 \mathrm{~g}$ Reparationsstadium nach Kolitis.

20. J. L. 3 Mon. A 3200 B 10 Tage E $3300 \quad$ T.Z. $10 \mathrm{~g}$ Pertussis. Temperatur meist zwischen $38,0-39,0^{\circ}$.

Dekomposition, parenteraler Infekt.

$\begin{array}{llllll}\text { 21. F. L. } & 91 / 2 \text { Mon. A } 6120 & \text { B } 27 \text { Tage } & \text { E } 7000 & \text { I'.Z. } 32 \mathrm{~g}\end{array}$ Milchschorf.

$\begin{array}{llllll}22 . & \text { A. H. } 6 \text { Mon. A } 3700 & \text { B } 22 \text { Tage E } 4800 & \text { T.Z. } 50 \mathrm{~g}\end{array}$ Ausgedehnte Furunculose. Repariertes Kind.

23. B. S. 3 Mon. A 2900 B 12 Tage E 3100

T.Z: $16 \mathrm{~g}$

Parenteraler Infekt. Otitis und Bronchitis.

24. E. K. 1 Mon. A $2700 \quad$ B 9 Tage E $2900 \quad$ T.Z. $22 \mathrm{~g}$ Ekzem.

25. A. R. 3 Mon. A 2700 B 15 Tage E $3200 \quad$ T.Z. $33 \mathrm{~g}$ Ruhrartige Erkrankung in Reparation.

$\begin{array}{llllll}\text { 26. } & \text { E. H. } & 4 \text { Mon. A } 5000 & \text { B } 12 \text { Tage E } 5400 & \text { T.Z. } 33 \mathrm{~g}\end{array}$ Genesenes Kind.

$\begin{array}{llllll}\text { 27. E. St. } 7 \text { Mon. A } 4300 & \text { B } 28 \text { Tage } & \text { E } 4900 & \text { T.Z. } 21 \text { g }\end{array}$ Mehlnährschaden, Dekomposition 2. Grades.

28. H. O. 2 Mon. A 3640 B 23 Tage E $4200 \quad$ T.Z. $19 \mathrm{~g}$ Reparationsstadium nach Dekomposition. 
29. B. Sch. 6 Mon. A $5700 \quad$ B 9 Tage E $6000 \quad$ T.Z. $33 \mathrm{~g}$ Gesundes Kind.

$\begin{array}{llllll}\text { 30. K. Sp. } 6 \text { Mon. A } 5200 & \text { B } 14 \text { Tage E } 5800 & \text { T.Z. } 43 \mathrm{~g}\end{array}$ Reparation nach Dekomposition.

$\begin{array}{llllll}\text { 31. F. D. } & 8 \text { Mon. A } 4800 & \text { B } 27 \text { Tage } & \text { E } 5600 & \text { T.Z. } 30 \mathrm{~g}\end{array}$ Pertussis.

32. H. W. 7 Mon. A 4650 B 21 Tage E $5150 \quad$ T.Z. $24 \mathrm{~g}$ Reparationsstadium nach Kolitis.

33. A. R. 3 Mon. A $2720 \quad$ B 17 Tage E $3300 \quad$ T.Z. $34 \mathrm{~g}$ Parenterale Infektion. Bilanzstörung.

$\begin{array}{llllll}\text { 34. Ph. H. } 5 \text { Mon. A } 5600 & \text { B } 19 \text { Tage } & \text { E } 6100 & \text { T.Z. } 27 \mathrm{~g}\end{array}$ Bilanzstörung.

$\begin{array}{llllll}\text { 35. G. H. } 4 \text { Mon. A } 3900 & \text { B } 28 \text { Tage } & \text { E } 4550 & \text { T.Z. } 23 \mathrm{~g}\end{array}$ Dystrophie.

36. K. R. 8 Mon. A 4020 B 22 Tage E $4300 \quad$ T.Z. $12 \mathrm{~g}$

Pertussis. Temperaturen zwischen 38 und 39 , dabei Gewichtssturz um $400 \mathrm{~g}$, dann ansteigende Grewichtskurve.

37. E. M. H. 6 Mon. A 5300 B 11 Tage E $5440 \quad$ T.Z. $12 \mathrm{~g}$ Parenteraler Infekt.

$\begin{array}{llllll}\text { 38. D. J. } 8 \text { Mon. A } 5020 & \text { B } 13 \text { Tage E } 5220 & \text { T.Z. } 15 \mathrm{~g}\end{array}$ Pertussis.

39. J. H. 10 Mon. A 3100 B 38 Tage E $3500 \quad$ T.Z. $11 \mathrm{~g}$ Dekomposition II. Rachitis, Pertussis.

40. R. P. 2 Mon. A 3700 B 3 Tage E 3820 T.Z. $40 \mathrm{~g}$ Gesundes Kind.

41. D. J. 4 Mon. A 4300 B 14 Tage $\quad$ E $4600 \quad$ T.Z. $21 \mathrm{~g}$ Reparationsstadium nach Intoxikation. Speier.

42. J. E. 6 Mon. A $3200 \quad$ B 39 Tage E $4700 \quad$ T.Z. $38 \mathrm{~g}$ Rachitis, Inanition.

43. M. H. 7 Mon. A 4100 B 6 Tage E $4300 \quad$ T.Z. $33 \mathrm{~g}$ Reparation nach akuter Dyspepsie und Bronchitis.

44. B. H. 3 Mon. A 3800 B 6 Tage E $3900 \quad$ T.Z. $16 \mathrm{~g}$ Reparation nach akuter Dyspepsie.

45. M. M. 21/2 Mon. A 3000 B 18 Tage E $3500 \quad$ T.Z. $27 \mathrm{~g}$ Parenterale Infektion.

46. E. Sch. 2 Mon. A $3600 \quad$ B 31 Tage E $4320 \quad$ T.Z. $23 \mathrm{~g}$ Bilanzstörung.

47. A. W. 3 Mon. A 5600 B 7 Tage E $6000 \quad$ T.Z. $55 \mathrm{~g}$ Hasenscharte, Inanition, sonst gesundes Kind.

48. J. A. 6 Mon. A 3500 B 29 Tage E $4200 \quad$ T.Z. $24 \mathrm{~g}$ Dekomposition I, Rachitis.

49. H. W. 4 Mon. A 5000 B 43 Tage E $6850 \quad$ T.Z. $43 \mathrm{~g}$ Gesundes Kind (nach Inanition). 
Die pathogenetische Bedeutung der Fettsäuren in Fettmilch-Nahrungen. 195

50. F. M. 5 Mon. A 4500 B 19 Tage E $4700 \quad$ T.Z. $10 \mathrm{~g}$ Chronische Dyspepsie. Parenterale-Infektion. Stühle meist zerfahren oder sehleimig. Bei Umstellung auf B.M.N. wird Gewichtskurve steiler.

$\begin{array}{llllll}\text { 51. A. R. } & \text { 6 Mon. A } 3800 & \text { B } 13 \text { Tage E } 4050 & \text { T.Z. } 19 \mathrm{~g}\end{array}$ Akuto Dyspepsie. Parenterale Infektion. Otitis media, Stühle bei saurer B.M.N. meist zerfahren, sehleimig.

52. M. Sch. 3 Mon. A $3890 \quad$ B 16 Tage E $4260 \quad$ T.Z. $23 \mathrm{~g}$ Speier. Gutes Gedeihen bei B.M.N. (sauer) und Brei.

53. K. G. 3 Mon. A 4460 B 11 Tage E $4920 \quad$ T.Z. $41 \mathrm{~g}$ Universelles Ekzem. Keine Verschlimmerung des Ekzems.

54. K. L. $71 / 2$ Mon. A $4680 \quad$ B 9 Tage $\quad$ E $4900 \quad$ T.Z. $26 \mathrm{~g}$ Abscesse am Kopf. 4 Tage lang Temperatur von 38,9 . Gutes Gedeihen.

$\begin{array}{lllll}\text { J5. A. G. } & 1 \frac{1}{2} \text { Mon. A } 5100 \quad \text { B } 15 \text { Tage E } 5600 \quad \text { T.Z. } 33 \mathrm{~g}\end{array}$ Neigung zu Ekzemen, Neuropath. Gute Stühle.

$\begin{array}{llllll}\text { 56. J. E. } 7 \text { Mon. A } 3200 & \text { B } 39 \text { Tage E } 4750 & \text { T.Z. } 39 \mathrm{~g}\end{array}$ Rachitis, Hydrocephalus. Die ersten 2 Wochen normale Temperatur, sehr gutes Gedeihen. Dann infolge parenteraler Infektion einige Temperaturzacken. Auch jetzt geht Kind gut.

57. L. M. G. 6 Mon. A 3900 B 15 Tage E $4500 \quad$ T.Z. $40 \mathrm{~g}$ In Reparation nach parenteraler Infektion.

58. R. M. 4. Mon. A $3480 \quad$ B 10 Tage E $3800 \quad$ T.Z. $32 \mathrm{~g}$ Rachitis, Bilanzstörung.

59. R. G. 2 Mon. A 1800 B 9 Tage E $1900 \quad$ T.Z. $10 \mathrm{~g}$ Enterale Infektion (Fruihgeburt).

60. W. M. 3 Mon. A $3350 \quad$ B 14 Tage E $4000 \quad$ T.Z. $46 \mathrm{~g}$ Reparation nach parenteraler Störung (Bilanzstörung).

Nicht gedeihende Kinder bei saurer Bnttermehlnahrung.

61. K. I. 2 Mon. A 3180 B 36 Tage E $3300 \quad$ T.Z. $3 \mathrm{~g}$

Phlegmonöses Erysipel. Über 4 Wochen anhaltende septische Temperaturkurve, Fieber bis zu $40^{\circ}$. Stuhl breiig.

62. F. D. $43 / 4$ Mon. A $3800 \quad$ B 17 Tage E $3800 \quad$ T.Z. Ruhr, Bronchopneumonie. Temperaturen meist $38^{\circ}$. Stühle geformt.

63. F. D. 6 Mon. A $3600 \quad$ B 5 Tage E $3400 \quad$ T.Z. $-40 \mathrm{~g}$ Ruhrrezidiv, 'lemperatur bis $39,0^{\circ}$.

64. B. Sch. 6 Mon. A $4600 \quad$ B 16 Tage F $4750 \quad$ T.Z. $9 \mathrm{~g}$ Parenterale Infektion.

65. J. D. 1 Mon. A $2800 \quad$ B 7 Tage E $2800 \quad$ T.Z. Speier. Erbrechen nach jeder Mahlzeit. Bei saurer B.M.N. zunächst Gewichtsstillstand. Bei Umstellung auf süße B.M.N. Gewichtssturz um $400 \mathrm{~g}$; das Gewicht bleibt auf Umstellung auf Frauenmilch bzw. Casein bis zum Exitus (3 Wochen später) stehen.

66. A. G. 12 Mon. A 5300 B 50 Tage E 5300 T.Z. -

Schwere Rachitis, chronische Bronchopneumonie (Bronchiektasie). Spasmophilie. Stets Temperatur über $38,0^{\circ}$. 
67. A. M. 6 Mon. A 5000 B 13 Tage E $4900 \quad$ T.Z. $-7 \mathrm{~g}$ Parenterale Infektion. Temperatur meist $38,0^{\circ}$. Zunächst bei saurer B.M.N. keine Tendenz zum Ansatz, die auch bei Umstellung auf süße B.M.N. nicht eintritt.

$\begin{array}{llllll}\text { 68. J. N. } & 5 \text { Mon. A } 3150 & \text { B } 17 \text { Tage E } 2820 & \text { T.Z. }-19 \mathrm{~g}\end{array}$

Parenterale Infektion, Mehlnährschaden. Gute Stühle, bei saurer B.M.N. stete Gewichtsabnahme, auch bei Umstellung auf Caseinmilch. Nach 4 Wochen Exitus.

69. F. W. $4 \frac{1}{2}$ Mon. A $3600 \quad$ B 7 Tage E $3600 \quad$ T.Z. -

Dekomposition II. Bei guten Stühlen und normaler Temperatur unter saurer B.M.N. keinerlei Ansatz, der später auch bei Zwiemilch ausbleibt. Gewichtsabfall bis zum Exitus nach 3 Wochen.

$\begin{array}{llllll}\text { 70. P. G. } 6 \text { Mon. A } 4600 & \text { B } 12 \text { Tage } & \text { E } 4700 & \text { T.Z. } 8 \mathrm{~g}\end{array}$ Lungentuberkulose. Beiderseits. Temperatur immer nahe $38,0^{\circ}$. Pirquet + .
71. L. M. G. 1 Mon.
A 2100 .
B 15 Tage E 2080
T.Z. $-1,5 \mathrm{~g}$ Parenterale Infektion. Speier.

72. H. A. 13 Mon. A 5500 B 7 Tage E $5350 \quad$ T.Z. $-7 \mathrm{~g}$ Ausgangsstadium einer serösen Meningitis? Encephalitis? Später Exitus. Obduktion verweigert.

73. E. R. 4 Mon. A 5900 B 18 Tage E $6000 \quad$ T.Z. $5 \mathrm{~g}$ Parenterale Infektion (Bronchitis). Stühle in Ordnung.

Aus diesen klinischen Erfahrungen geht mit Sicherheit hervor, daß auch viele ernährungsgestörte Kinder, besonders solche mit parenteraler Infektion, ja solche mit septischen Zuständen diese saure Buttermehlnahrung ausgezeichnet vertrugen. Nicht ein einziges Kind zeigte einen Schaden, den wir direkt a uf die Säuerung der Milch zurückführen konnten. Ähnliche Erfahrungen haben Nieman $n^{1}$ ) einerseits und Lange ${ }^{2}$ ) an der Klotzschen Klinik andererseits gemacht, die bei Ersatz der Milch durch Buttermilch nicht nur schlechtere, sondern zum Teil auch bessere Erfolge sahen und gesäuerte Buttermehlnahrung ist gewissermaßen nichts anderes als Buttermilch mit Butter und Kohlenhydraten versetzt. Bei einzelnen Kindern gewinnt man tatsächlich den Eindruck, daß die saure Buttermehlnahrung der süßen überlegen ist. Daß einzelne Kinder bei dieser Nahrung abnehmen, ja daß sie für einzelne nicht geeignet war, darf nicht wundernehmen; besteht doch darüber nicht der geringste Zweifel, daß auch die süße Buttermehlnahrung nichts weniger wie indifferent ist und somit Schädigungen machen kann (Lange u. a.). Es ist bei der spontanen Säuerung der Milch völlig gleichgültig, ob in der Milch dabei Fett anwesend ist oder nicht, da die Mengen der freien Fettsäuren, die in der Milch bei der gewöhnlichen im Haushalt vorkommenden Säuerung sich bilden, einmal nur aus dem Zucker entstehen und quantitativ außerordentlich gering sind ${ }^{3}$ ).

1) Niemann und Foth, Jahrb. d. Kinderheilk. 93, 137. 1920.

2) Lange, Zeitsehr. f. Kinderheilk. 22. 1919.

3) Dabei bleibt die Tatsache vollkommen unberührt, daß es ein Optimum der 
Um so auffallender ist es und in stärkstem Gegensatz zu unseren Beobachtungen stehend sind die Ergebnisse, die Berendi) jüngst in Budapest mit gesäuerter Milch machte. Bei Verfütterung der Buttermehlnahrung sah er bei drei von denen zur Zeit in Behandlung gestellten Kindern mehrere Tage blutig-eitrige Stühle, sobald er die Milch der Buttermehlnahrung durch saure Milch ersetzte. Leider gibt er hier keine genaue Mitteilung dieser wichtigen Fälle. Später hat er nun Schweinefett stats der Butter verwendet. Er gibt dabei an, daß die Erfolge mit dieser Fettmehlsuppe nicht die gleich guten waren wie die bei der Buttermehlsuppe. Doch war er im großen und ganzen zufrieden. Bcrend wollte nun erproben, ob die Budapester Kollegen auch im Sommer, wenn die Milch sauer wird, solche Fettmehlsuppe mit saurer Milch geben könnten, und dazu machte er folgendes Experiment. Er schreibt: ,Um dies erst mit sonst einwand freier ${ }^{2}$ ) Milch zu erproben, gab ich 6 älteren Säuglingen, welche bei Fettmehlernährung schon seit Wochen tadellos gediehen, eine solche Fettmehlsuppe, in welcher die zugesetzte Drittelmilchmenge bis $10 \mathrm{~g}$ Soxhlet-Henkel im Krankenhaus angesäuert wurde ${ }^{4 / 3}$ ). Aus diesem Satz könnte man nun folgern, daß hier nicht spontan gesäuerte Milch verwendet wurde, sondern die verwendete Milch durch Zusatz irgendeiner Säure bis $10 \mathrm{~g}$ Soxhlet angesänert wurde.

Diese angesäuerte Milch, die aber natürlich ganz etwas anderes darstellt wie spontan gesäuerte, zumal sie auch noch Schweinefett und nicht Butter enthält, das als Fett für den Säuglingsdarm noch weniger adäquat ist als Butter, vertrugen nur 2 Kinder ohne Krankheitssymptome. Bei 2 Kindern stellten sich Durchfälle ein und 2 Kinder reagierten mit schwersten Zuständen blutig-schleimiger Entleerung, also ruhrähnlichen Erscheinungen. Die beiden letzten Kinder starben. Bei der Sektion typische Enteritis dysenteriformis. Bakteriologisch Ruhrbacillen negativ. Später hat Berend diese gesäuerte Nahrung mit $\mathrm{CaCO}_{3}$ versetzt und seitdem nie wieder solche Zufälle gesehen. Ein direkter Zusammenhang zwischen der Nahrung und der schweren Darmerkrankung ist sehr wohl möglich und soll nicht in Abrede gestellt werden. Aber entschieden ist zu bestreiten, daß das, was Berend hier gesehen hat, eine Folge der Säuerung der Milch ist. Einmal hat er Schweinefett zur Nahrung verwendet, über das alle Erfahrungen bei

Säuerung gibt. So löst z. B. se hr sa u re Milch, sei sie Fettmilch oder Buttermileh, leichter Erbrechen aus. Diese Tatsache hat aber praktisch wenig Bedeutung, da eine solche saure Milch wohl selten verfüttert wird. Und wir halten es für absolut falsch, diese Tatsache für die Pathogenität saurer Milch heranzuziehen, eben weil sie den wirklichen Verhältnissen nicht gerecht wird.

1) Berend, Monatssehr. f. Kinderheilk. 14, 601 .

2) Auch im Original gesperrt gedruckt.

3) Im Original nicht gesperrt gedruckt. 
Säuglingen fehlen, und sodann gibt er als Säuregrad 10 Henkel-Soxhlet an, einen äußerst geringen Grad. Endlich ist aber das klinische Bild, unter dem die Kinder erkrankten, ein völlig anderes als das der alimentären Intoxikation. Was Berend beschreibt und was anatomisch sich auch nachher herausstellt, ist das Bild der dysenterieformen Kolitis, wie sie meist auf dem Boden einer echten enteralen Infektion entsteht, gleichviel, ob dabei echte Ruhrbacillen verantwortlich zu machen sind oder nicht. Jedenfalls liegen hier so abwegige Verhältnisse vor, daß keinesfalls diese Fälle als Beweise gegen die Gefährlichkeit einer Säurebildung aus Fett ins Feld geführt werden können ${ }^{1}$ ).

Wir haben übrigens solche Schweinefettnahrungen hergestellt und nach 24stündigem Stehenlassen und Ansäuern folgende Mengen freier Fettsäuren in jener Fettmehlnahrung nach Berend gefunden:

\begin{tabular}{|c|c|c|c|}
\hline $\begin{array}{l}\text { Schweinefettmehl- } \\
\text { nahrung }\end{array}$ & $\begin{array}{l}\text { Flüchtige } \\
\text { in } 1 / 10 \mathrm{NaOH} \\
\text { in } 100 \mathrm{~g}\end{array}$ & $\begin{array}{l}\text { Fettsäuren } \\
\begin{array}{r}\text { in } 100 \mathrm{~g} \\
\text { essigsaure }\end{array}\end{array}$ & - $\begin{array}{c}\text { Gesamte Aci- } \\
\text { dität } \\
\text { Henkel-Soxhiet }\end{array}$ \\
\hline & 3,0 & 0,018 & 16,0 \\
\hline
\end{tabular}

Fütterungsversuche mit Fettmehlnahrung haben wir nicht gemacht. Es geht vielmehr aus unseren Versuchen und unseren klinischen Erfahrungen hervor, daß nicht die in der Milch präformierten Fettsäuren es sind, die schädlich wirken, sondern da $\beta$ diese schädliche Wirkung der Kuhmilch erst im Darm bei pathologischen Verhältnissen zutage tritt. Selbst in der sauren, reichlich mit Fett versetzten Nahrung ist die Menge dieser Säuren sehr gering und kommt vor dem GenuB wesentlich nur durch Spaltung des Zuckers, nicht

1) Nach Fertigstellung des Manuskripts teilt mir Herr Kollege Heim, der nach dem tragisehen Tode des verdienten ungarischen Forschers das Spital Berends übernommen hat, auf briefliche Anfrage folgendes mit: Eine Assistentin Berends ist noch in der Klinik tätig und berichtet darüber folgendes. Eine Ansäuerung der Milch durch Zugabe einer freien Fettsäure (Essigsäure) hat nicht stattgefunden. Damit entfällt unsere obige Vermutung. Die Milch wurde aufgekocht und dann 24 Stunden stehen gelassen, um zu säuren. Der Effekt der Fütterung war geradezu, ,katastrophal". 17 Kinder erkrankten zum Teil sehr schwer, zum Teil starben sie, also war die Sache noch weit schlimmer als Berend mitteilt. . Eine Erklärung vermag auch Heim nicht zu geben. Er teilte aber mit, daß viele schwere enterale und parenterale Infektionen im Hause herrschten und dadurch vielleicht eine Infektion der Milch hervorgerufen sei.

Es ist in der Tat sehr schwer, besonders da der Autor nicht mehr lebt, diese Beobachtungen nachträglich richtig zu deuten. Zweifellos können es nicht die Säuren gewesen sein, zumal der Säuregrad nur 10 Henkel-Soxhlet betrug, also sehr gering ist. Das wahrscheinlichste ist mir - dafür spricht auch das klinische Bild -, daß es sich um den seltenen Fall einer echten Milchvergiftung handelt, also um eine Art Typhus oder Paratyphusvergiftung. Restlos ist die Frage heute nicht zu klären. 
des Fettes, zustande. Denn saure Fettmischungen enthalten nicht mehr freie Fettsäuren als saure Magermilch (Buttermilch). Die freien Fettsäuren, die im Magen entstehen, stammen wohl aus dem Fett, sei es durch fermentative Abspaltung, sei es durch bakterielle Prozesse. Sie sind bei saurer Nahrung vielleicht etwas höher, sind aber immer noch so gering, so daß ihnen praktisch keine wesentliche pathologische Bedeutung zukommt. Auch das wird durch die klinische Erfahrung bewiesen. Andere bakterielle Gifte, die durch Saprophyten in der Milch-entstehen, sind noch weniger nachgewiesen und spielen bei der spontanen Säuerung überhaupt keine Rolle, da die anderen Keime durch die Säurebildner in ihrer Entwicklung gehemmt werden.

Immer wieder muß man, wenn man sachlich diese Frage erörtert, hören, daß es gefährlich sei, ,die mühsam errungenen Forderungen der Milchhygiene" so zu diskreditieren (Thiemich). Davon kann keine Rede sein. Freilich die übertriebene Bakterienangst der Milch bekämpfen wir, aber ich glaube, daß es tatsächlich keinen anderen Weg gibt, als daß man sich in einer wissenschaftlichen Zeitschrift so darüber ausdrückt, wie ich es getan habe; denn ich betonte ausdrücklich, daß ,es mir himmelweit fern liege, daß etwa die Milchhygiene falle". Prinzipiell haben diese Dinge mit der Milehhygiene wirklich nichts zu tun. Eine verschmutzte und unappetitliche Milch bleibt verschmutzt und unappetitlich, auch wenn sie tatsächlich unschädlich wäre, gleichviel, ob sie sauer oder süß, roh oder gekocht oder sonstwie genossen wird. Wir dürfen gesundheitsschädlich nicht mit ekelerregend und unappetitlich verwechseln. Man überlasse es dem Laien darüber zu diskutieren, daß schlecht schmeckende oder im Geschmack veränderte Speisen auch schädlich sein müssen und folge den Wegen, die uns naturwissenschaftlich immer noch am weitesten bringen: den klinischen Erfahrungen und dem Experiment. Und diese sprechen für die gute Bekömmlichkeit spontan gesäuerter Milch beim Säugling. Wir haben dabei stets die geronnene Milch gut gequirlt, so daß das Casein feinflockig zur Gerinnung kam und halten dies für wichtig, weil auch die Milch dadurch besser durch den Sauger getrunken werden kann. Endlich erscheint uns praktisch wichtig noch die Frage, ob nicht ein Zusatz von Alkali am Platze ist. Länger eine Milch alkalisch zu halten, erscheint uns bedenklich; dadurch werden peptonisierende Bakterien die Uberhand gewinnen, und selbst wenn sie nicht pathogen sein mögen, so würde sich doch bald durch einen intensiven Käsegeruch eine Zersetzung des Eiweißes bemerkbar machen und die Milch würde „ungenießbar und ekelerregend". Sie braucht deshalb noch lange nicht giftig zu wirken; denn solche Käsemilch wird im allgemeinen noch gut vertragen. Aber sicher ist diese Milch ekelerregend und dürfte als absolut ungenießbar an- 
200 Rietsehel: Die pathogenetische Bedentung der Fettsäuren แลพ.

gesehen werden. Deshalb habe ich mich stets gegen den Zusatz von Natron zur Milch in Molkereien ausgesprochen, weil dadurch diesen peptonisierenden Prozessen außerordentlich leicht Vorschub geleistet wird. Wiederholt haben uns Mütter Milchproben gebracht, die Seifenoder Käsegeruch aufwiesen. Es ist zweifellos zweckmäßig, lieber die Milch ansäuern zu lassen, aber dann den Müttern zu empfehlen, wenn ihnen die saure Milch irgendwie bedenklich erscheint, sie vor dem Trinken zu alkalisieren. Dazu möchten wir nach dem Vorşchlage von Berend das $\mathrm{CaCO}_{3}$ verwenden. Nötig ist es nicht. Es werden vielleicht einmal Zeiten kommen, wo die Milchknappheit eine so große ist, daß wir jeden Tropfen dem jungen Kinde zufließen lassen müssen. Jedenfalls ist nach unseren Erfahrungen schon heute bei dem hohen Milchpreis auszusprechen, daß Anstalten ruhig spontan gesäuerte Milch zur Fütterung von Säuglingen verwenden sollten und weitere Erfahrungen sammeln.

Die saure Milch als allgemeine Volksnahrung den Müttern für ihre Kinder zu empfehlen, wird man aus praktischen Gründen heute noch nicht zustimmen können, wohl aber liegt kein Grund vor, Konservenmilch für den Sommer zu empfehlen, um den Gefahren einer sommerlichen bakteriellen Milchverderbnis ${ }^{1}$ ), die unseres Erachtens praktisch keine entscheidende Rolle spielt, zu entgehen. Letzten Endes schützt uns auch nicht die Konserve vor Milchver giftungen, denn es ist bekannt, daß gerade von Konserven aus Nahrungsmittelvergiftungen ausgehen können (Fleisch, Gemüse usw.).

1) Neuland u. Peiper, Med. Klin. Nr. 47, S. 1199.1920.

Würzburg, Universitäts-Kinderklinik. 